\title{
Application of a patient-derived xenograft model in cytolytic viral activation therapy for nasopharyngeal carcinoma
}

\author{
Cheng-Lung $\mathrm{Hsu}^{1}$, Yung-Chia Kuo ${ }^{1}$, Yenlin Huang ${ }^{2}$, Yin-Cheng Huang ${ }^{3}$, \\ Kar-Wai Lui ${ }^{4}$, Kai-Ping Chang ${ }^{5}$, Tung-Liang Lin ${ }^{1}$, Hsien-Chi Fan ${ }^{1}$, An-Chi Lin ${ }^{1}$, \\ Chia-Hsun Hsieh ${ }^{1}$, Li-Yu Lee ${ }^{2}$, Hung-Ming Wang ${ }^{1}$, Hsin-Pai Li ${ }^{6}$, Yu-Sun Chang ${ }^{6}$ \\ ${ }^{1}$ Division of Hematology-Oncology, Department of Internal Medicine, Chang Gung Memorial Hospital, Chang Gung University, \\ Taoyuan 333, Taiwan, ROC \\ ${ }^{2}$ Department of Pathology, Chang Gung Memorial Hospital, Chang Gung University, Taoyuan 333, Taiwan, ROC \\ ${ }^{3}$ Division of Neurologic Surgery, Department of Surgery, Chang Gung Memorial Hospital, Chang Gung University, Taoyuan \\ 333, Taiwan, ROC \\ ${ }^{4}$ Department of Medical Imaging and Intervention, Chang Gung Memorial Hospital, Chang Gung University, Taoyuan 333, \\ Taiwan, ROC \\ ${ }^{5}$ Department of Otolaryngology-Head and Neck Surgery, Chang Gung Memorial Hospital, Chang Gung University, Taoyuan \\ 333, Taiwan, ROC \\ ${ }^{6}$ Department of Cell and Molecular Biology, Chang Gung University, Taoyuan 333, Taiwan, ROC
}

Correspondence to:

Cheng-Lung Hsu, e-mail: hsu2221@adm.cgmh.org.tw

Keywords: NPC, PDX, EBV, target therapy, viral lytic therapy

Received: July 09, $2015 \quad$ Accepted: September 11, 2015

Published: September 24, 2015

\section{ABSTRACT}

\begin{abstract}
Nasopharyngeal carcinoma (NPC) is an Epstein Barr virus (EBV)-related malignancy in which the tumor microenvironment plays a pivotal role in tumor progression. Here, we developed two patient-derived xenograft (PDX) mouse lines from engrafted NPC metastatic tumors. Positive staining for EBV-encoded small RNAs confirmed that these tumors harbored EBV, and gene expression profile analyses further showed that the PDX was highly similar to the primary parent tumor. In vivo drug screening using the PDX system demonstrated that gemcitabine had the best antitumor effect among the tested drugs. The donor of this PDX also showed excellent responsiveness to gemcitabine treatment. The combination of gemcitabine and valproic acid exerted synergistic antitumor effects. Further addition of ganciclovir to this two-drug combination regimen enhanced cytolytic viral activation, yielding the best antitumor response among tested regimens. Treatment with this three-drug combination regimen decreased plasma EBV-DNA load, tumor viral concentration, and the number of viable tumor cells to a greater extent than the two-drug gemcitabine and valproic acid combination. These results highlight the value of PDX models in the development of EBV-targeted strategies to treat NPC.
\end{abstract}

\section{INTRODUCTION}

Nasopharyngeal carcinoma (NPC) is highly prevalent in Southern Chinese populations and has a predilection to affect young adult males [1]. In this endemic region, non-keratinizing and undifferentiated carcinoma constitutes up to $99 \%$ of all cases, and these tumors are closely related to infection with Epstein Barr virus (EBV) $[2,3]$. The presence of EBV in virtually all tumor cells highlights the integral relationship among virus, cancer cells, and tumor microenvironment [3]. In the past decade, the amount of free EBV-DNA in peripheral blood cells has been shown to correlate well with tumor stage, and has essentially become a standard marker for NPC [4]. EBV-DNA load has been identified as an independent prognostic factor in metastatic NPC patients such that those with a high pretreatment viral load have worse outcomes [5-7].

There has been a recent increase in the use of patient-derived xenografts (PDX) as a preclinical model 
[8]. In application, the tumor from a patient is directly implanted into a severe combined immunodeficiency (SCID) mouse and then re-implanted into subsequent passage mice to amplify the tumor mass. Several PDX models have been established. These models exhibit a stable biological profile when passaged in mice (especially during early passages) in terms of global gene-expression patterns, mutational status, metastatic potential, drug responsiveness, and tumor architecture [8]. Comprehensive genome-wide gene expression analyses have demonstrated that early passage PDX have key genomic expression profile features similar to those of primary tumors [9]. In head and neck cancer, the PDX model had been shown to harbor mutations in TP53 or exhibit amplification of CCND1 similar those observed clinically in association with cisplatin resistance $[10,11]$. Moreover, because orthotopic PDX models preserve a greater proportion of stromal components, they recapitulate tumor microenvironment effects, developing patterns of locoregional and distant metastases similar to those of human tumors $[12,13]$. Primary tumor xenografts have been shown to be valuable in a tailored personalized medicine setting for drug-sensitivity screening in cases where standard treatment has failed; they could also help to identify key pathway components suitable for targeted drug development [14].

The PDX model had been applied in NPC to study combinations of chemotherapy, radiotherapy and histone deacetylase (HDAC) inhibitors [15], and for characterizing the role of the microRNA, miR-31, in EBV-associated NPC [16].

In a cancer cell, EBV is in a latent phase and expresses 8-11 genes involved in maintaining EBV proliferation. Among the latent genes expressed in NPC, latent membrane protein 1 is considered the primary viral oncoprotein, facilitating tumor cell growth and local invasion, and conferring antiapoptotic properties and survival advantages [17]. EBV reactivation from latency requires expression of viral immediate-early transactivators of subsequent lytic genes, including thymidine kinase, protein kinase, and an EBV-encoded DNA polymerase. These gene products are essential for creating new viral genomes [18]. This EBV latent-lytic shift may offer a strategy for promoting EBV-dependent tumor cell killing. Some therapeutic agents, such as the HDAC inhibitor bortezomib, a variety of chemotherapy agents and irradiation, have been shown to induce the ATM-p53 pathway, which would promote EBV reactivation [19-23]. In lytic-induction strategies, some EBV-encoded kinases may convert nucleoside analogs such as ganciclovir into cytotoxic drugs that kill EBVpositive tumor cells and virus [20,24]. Furthermore, the phosphorylated form of ganciclovir can be transferred to adjacent tumor cells through gap junctions, resulting in "bystander killing" of a much greater percentage of tumor cells [20].

\section{RESULTS}

\section{Establishment and characterization of the EBV-positive PDX model}

Very few currently available NPC cell lines harbor endogenous EBV, which is important for NPC cell growth and progression. Furthermore, microenvironmentcancer cell interactions play a pivotal role in cancer cell progression in NPC. To address these issues, we sought to establish EBV-positive tumors via PDX in a SCID mouse model. Exploiting the fact that engrafting rate is higher for metastatic tumors than primary site tumors, we established two mouse xenograft lines from NPC metastatic tumors: NPC01, obtained from a paraspinal soft tissue tumor at initial diagnosis (before treatment) of NPC with bone and soft tissue metastasis, and NPC02, obtained from a neck lymph node biopsy after three lines of chemotherapy. Compared with the EBV-positive NPC cell line C666-1, a xenograft with a homogeneous single tumor cell type, PDX had a more heterogeneous histologic phenotype with various size tumor cells and background cells (Fig. 1). EBER staining confirmed that these tumors harbored EBV (Fig. 1). The PDX required a longer time to grow (3-6 months for one passage) than the cell line xenograft ( $\sim 2-3$ weeks from implantation of $5 \times 10^{5}$ cells to achieving a $1,000 \mathrm{~mm}^{3}$ xenograft).

An initial small biopsy/excisional tumor was amplified in the PDX system. An mRNA microarray analysis of these tumors compared with in vitro-cultured NPC cell lines and a C666-1 xenograft showed that the gene profile of the PDX tumor was more similar to the clinical sample than the C666-1 xenograft and was clearly different from that of in vitro-cultured EBV-positive and -negative cell lines (Fig. 2A). Expression levels of some hematopoietic/ macrophage-related genes were lower in the PDX than in the parent tumor, possibly reflecting the immunocompromised background of SCID mice. Because initial biopsy tissue pieces were small, we lacked sufficient parent human tumor for gene expression profile analysis of NPC02; however, the gene expression profile of this PDX line was still different from that of the C666-1 xenograft (Fig. 2B). Collectively, these results confirm that the PDX system reliably represents the clinical sample.

\section{Drug screening using an in vitro NPC cell line versus the in vivo PDX system}

After establishing this reliable tumor system, we used it to test sensitivities to the most commonly clinically used anticancer chemicals, using activity in in vitro cultured C666-1 cell line as a guide. Docetaxel, gemcitabine, and mitomycin-C showed good anticancer activity in the nano- to micromolar concentration range, although the $\mathrm{IC}_{50}$ for docetaxel was lower than that for gemcitabine and mitomycin-C (Fig. 3A). In contrast, cisplatin, fluorouracil, 

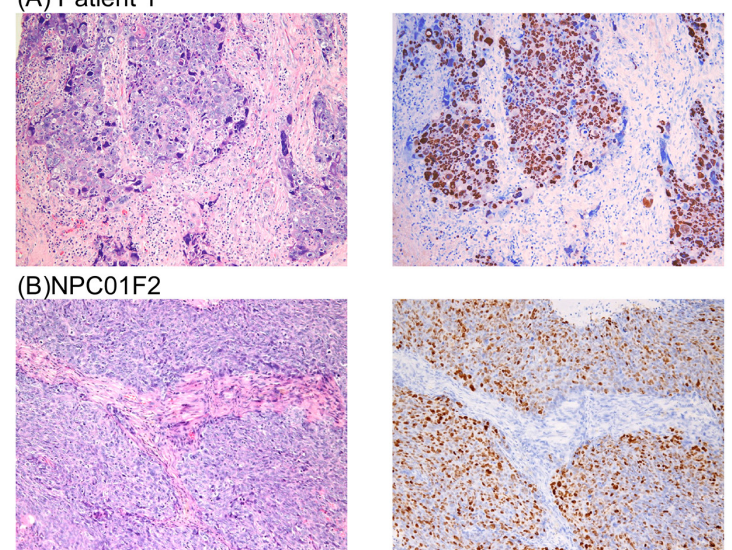

(C) Patient 2
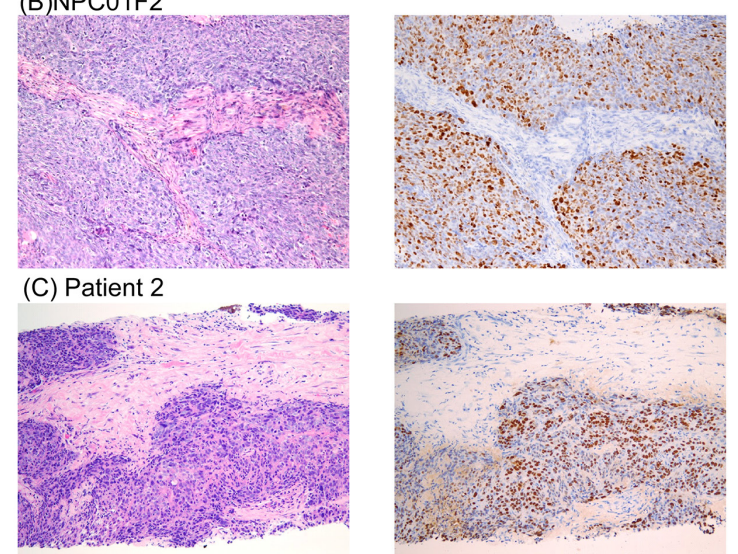

(D)NPC02F3
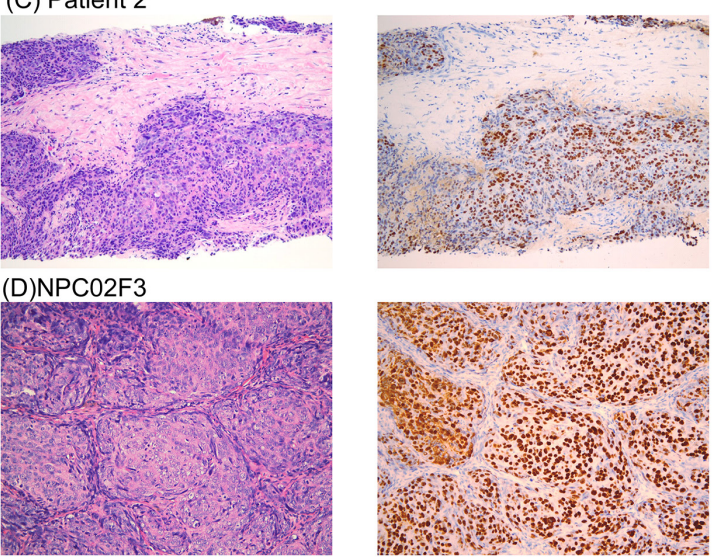

(E)C666-1
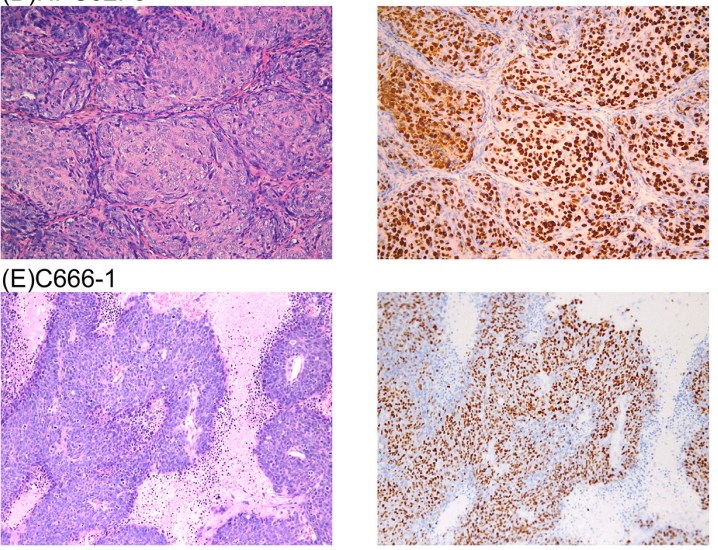

Figure 1: Histological comparison of the parent clinical tumor A, C. with PDX B, D. and NPC cell line (C666-1) xenografts E. H\&E staining (left column) and detection of EBER by in situ hybridization (right column). Original magnification, $200 X$.

etoposide, and valproic acid were largely ineffective except at higher concentrations (Fig. 3A). Notably, millimolar concentrations of valproic acid and ganciclovir were required to produce antitumor effects. We next tested these drugs in the PDX system. To our surprise, gemcitabine had the best anticancer effect among the tested drugs in the PDX model (Fig. 3B and 3C). Although cisplatin is effective in clinical practice, it was ineffective at the doses tested in the PDX system. Some diarrhea episodes were noted in the docetaxel treatment group. Mitomycin-C was also found to be an active drug in this in vivo assay (Fig. 3C).

\section{Drug sensitivity in PDX assays correlates with patient clinical response}

Patient number one died due to cancer progression before we were able to finish his drug-sensitivity screening in the PDX system. Gemcitabine had been shown to exert the best anticancer effect in our in vivo $\mathrm{NPC0} 2$ PDX system. Accordingly, we tested gemcitabine in patient number two, who had been treated unsuccessfully with five different anticancer treatments (Fig. 4A). Follow-up clinical assessments showed a decreased plasma EBV-DNA load (Fig. 4A) and diminished toxicity profile (not shown) in response to gemcitabine treatment, and an evaluation of drug response by bone scan showed stable disease (Fig. 4B and 4C).

\section{Application of the PDX model to cytolytic viral activation therapy}

Cytolytic viral activation therapy (CVAT), which shifts oncolytic viruses from the latent phase to the lytic phase and causes cancer cell death, has recently come to be considered a therapeutic strategy in EBV-related cancers, including lymphoma and NPC [25, 26]. Different 

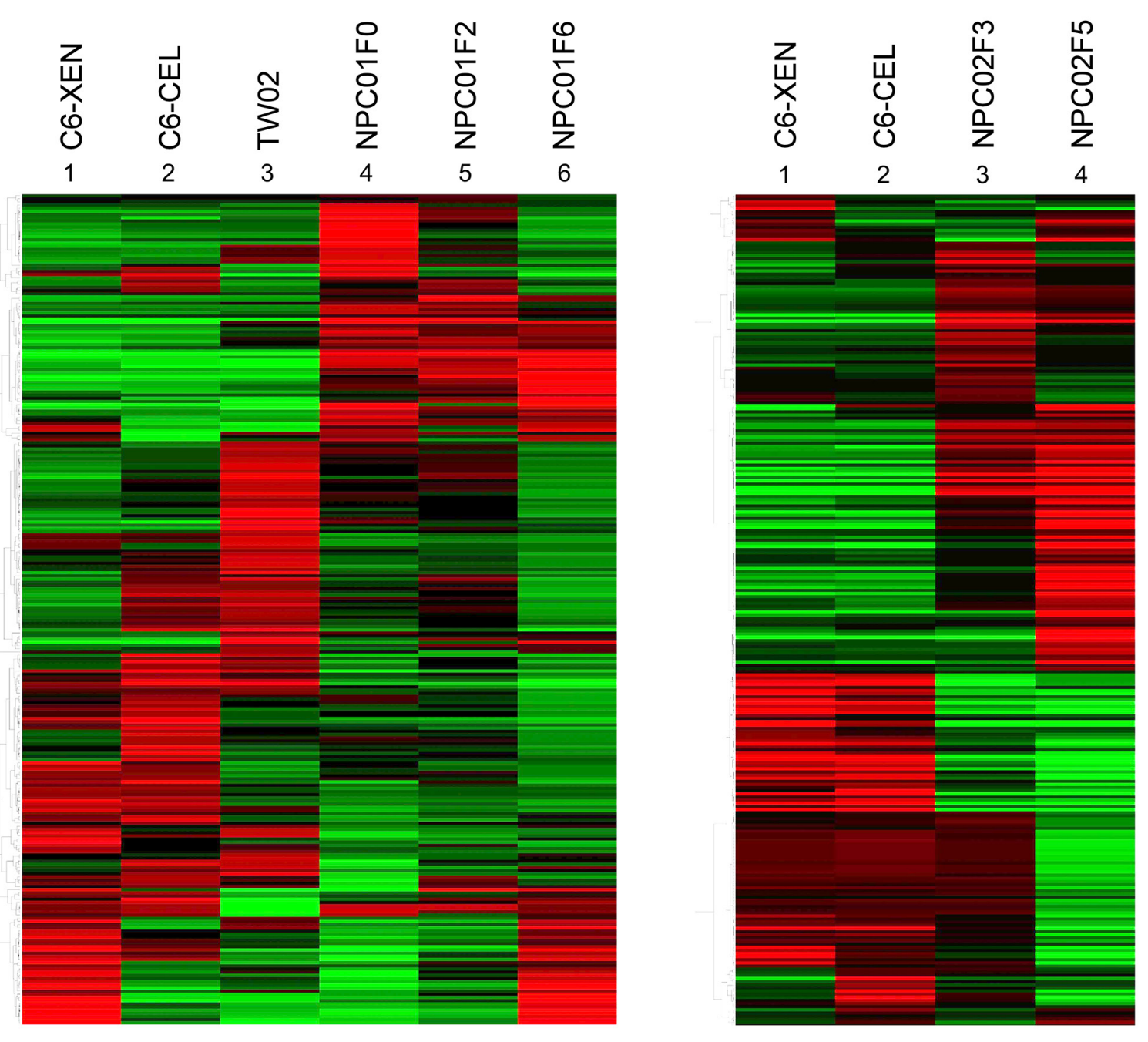

Figure 2: Comparison of gene expression profiles between clinical samples and PDX by microarray analysis. A. NPC01 was from a paraspinal soft tissue tumor obtained at initial diagnosis (before treatment) of NPC with bone and soft tissue metastasis. B. NPC02 was obtained from a small piece of neck lymph node biopsy after three lines chemotherapy; it could be amplified in the NOD/ SCID mice system, but a parent clinical sample was unavailable for microarray analysis. Abbreviations: C6-XEN, C666-1 xenograft; C6-CEL, C666-1 dish culture; TW02, NPC cell line, dish culture; NPC01F0, NPC patient 1 excision metastatic tumor; NPC01F2/NPC01F6, second and sixth passage PDX of NPC01F0; NPC02F3/NPC02F5, third and fifth passage PDX of NPC patient 2 neck lymph node biopsy.

combinations of standard chemotherapeutic agents, HDAC inhibitors, and antiviral agents have been reported to exert efficient anticancer activity toward lymphoma and NPC $[19,21,22,26]$. In initial tests of this concept in our PDX system, we found that, whereas gemcitabine alone effectively suppressed tumor growth, the combination of valproic acid and ganciclovir had no significant antitumor effect compared with DMSO controls (Fig. 5A, valproic acid + ganciclovir vs. gemcitabine). We confirmed the importance of the standard chemotherapeutic agent, gemcitabine, in these combination regimens by using a reduced dose of gemcitabine during the first 4 weeks of treatment, which produced little or no antitumor effect (Fig. 5B). A combination regimen consisting of gemcitabine and valproic acid exerted superior antitumor effects compared with gemcitabine alone (Fig. 5B, gemcitabine + valproic acid vs. gemcitabine). Further addition of ganciclovir to this gemcitabine + valproic acid regimen produced tumor control similar to that observed in the gemcitabine + valproic acid group (Fig. 5B, gemcitabine + valproic acid + ganciclovir vs. gemcitabine + valproic acid). Xenograft weights measured after sacrificing mice confirmed these tendencies (Fig. 5C).

Although adding ganciclovir had no additional tumorcontrolling effect, it is possible that it affected EBV-related activity. In support of this, we found that the ganciclovircontaining three-drug combination group not only had a lower plasma EBV-DNA load, but also a lower EBVDNA/tumor cell ratio in tumor tissue than the two-drug gemcitabine + valproic acid group (Fig. 6A and 6B). 
(A)
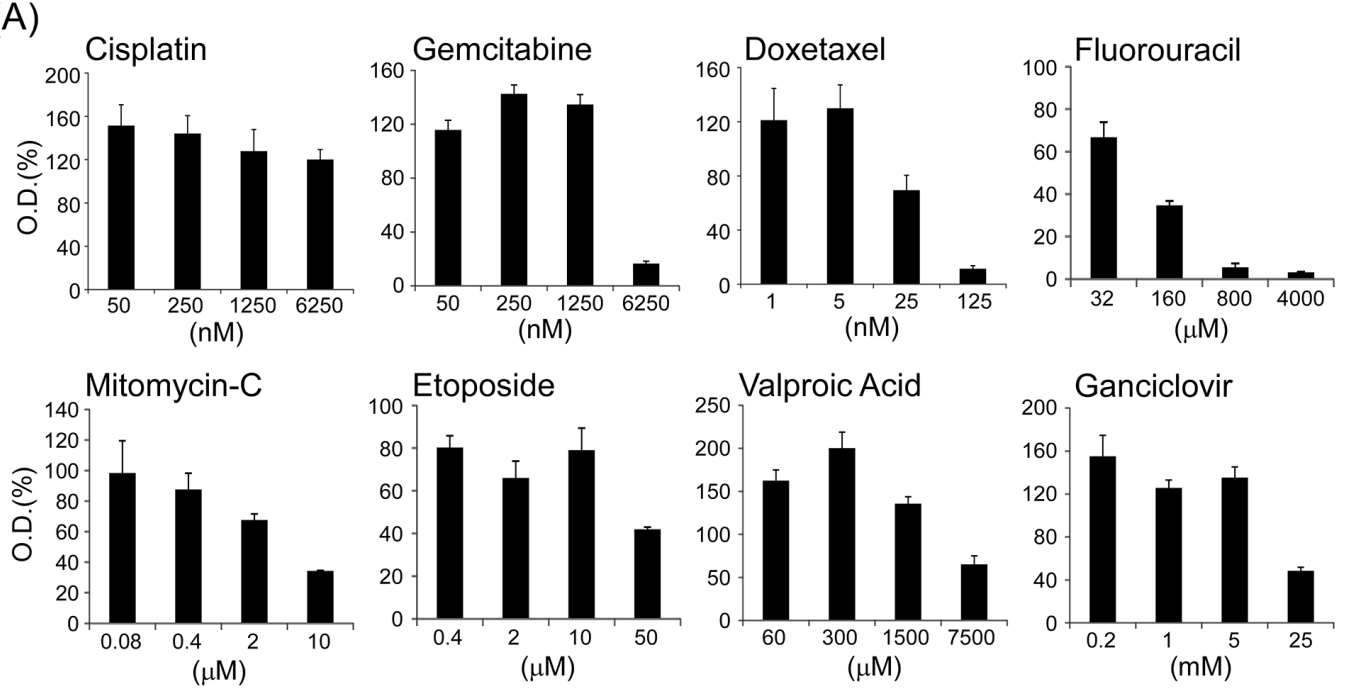

(B)
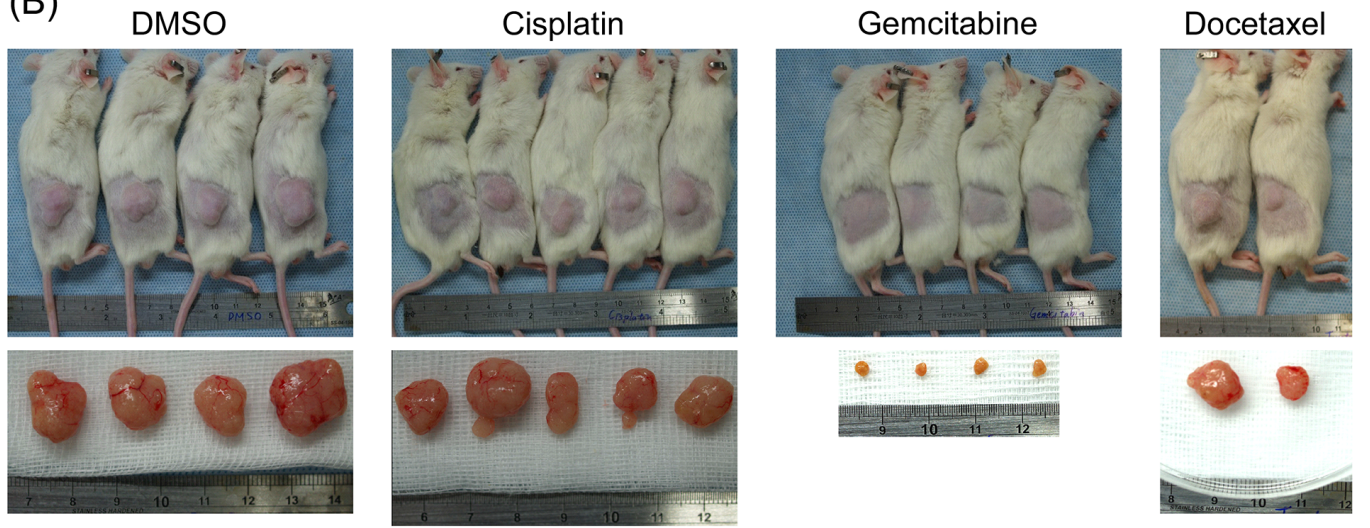

(C)

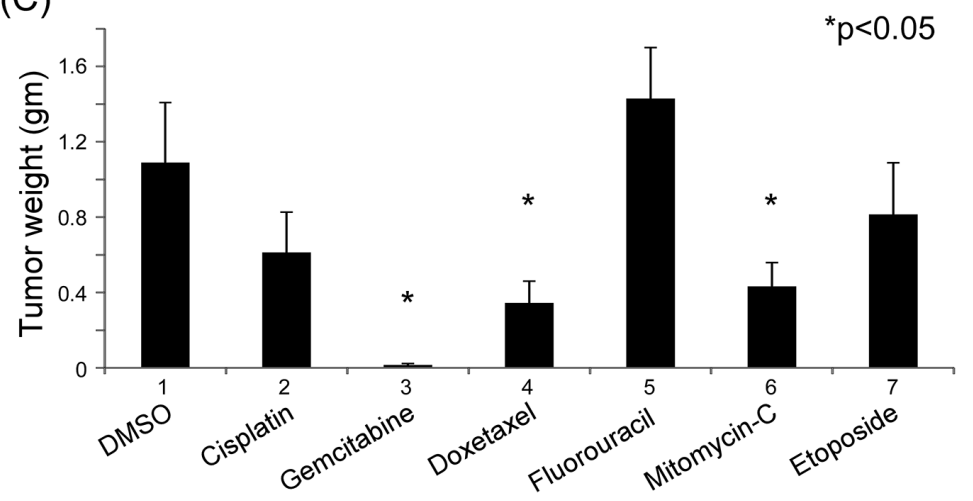

Figure 3: Drug-sensitivity screening in vitro using NPC C666-1 cell line growth assays A. and in vivo using the PDX model (B, C). A. C666-1 cells were plated at $5 \times 10^{4}$ cells/well in 24-well plates and incubated with or without different concentrations of tested drugs for 6 days. Cell growth was assessed using the MTT assay. Values presented in figures are mean $\mathrm{OD}_{590} \pm \mathrm{SD}$ from at least three independent reaction wells. B. NOD/SCID PDX subcutaneous tumor before and after excision. C. Xenograft tumor weight after sacrificing mice. After the tumor had been sub-implanted in NOD/SCID mice and the xenografts had reached a volume of approximately $150 \mathrm{~mm}^{3}$, animals were randomized (3-5 tumor-bearing mice per group) and various drug dose schedules, described in Materials and Methods, were administered via intraperitoneal injection. Mice were sacrificed 3 months after chemical injection or earlier in circumstances involving declining health status, morbundity, or unrelieved pain and discomfort. Tumor weights presented in figures are means \pm SD from at least three independent studies. The PDX used in these studies were from the first eight passages of each line. 
Nasopharyngeal carcinoma, T4N3bM1 with bone metastasis

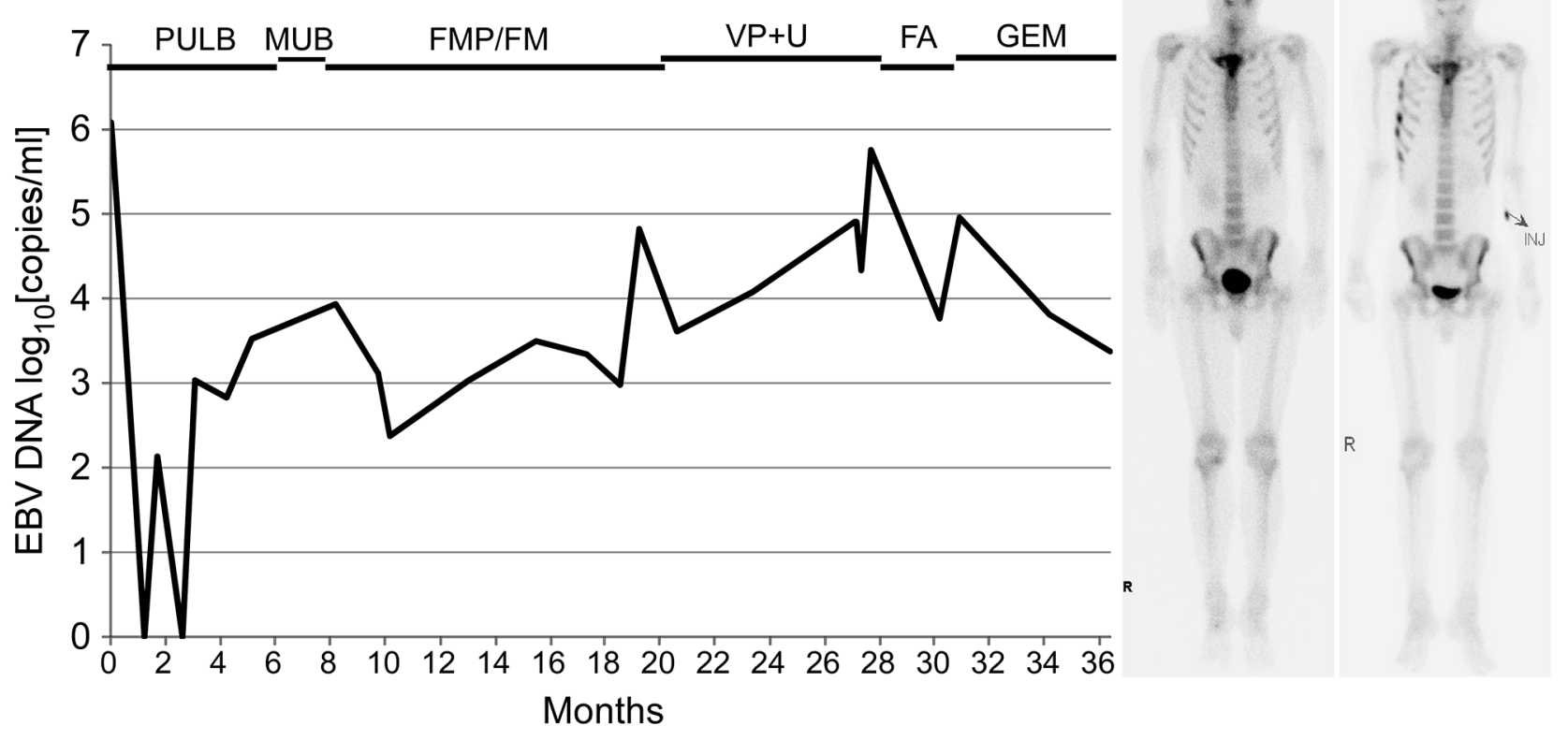

Figure 4: PDX drug sensitivity correlates with clinical response. NPC02, a 42 year-old NPC patient with bone metastasis, had received five different lines of palliative chemotherapy before gemcitabine (GEM) was prescribed. Allergy to cisplatin was found during treatment with the third chemotherapy regimen (FMP); thus, cisplatin was subsequently omitted. A. Plasma EBV-DNA load reflecting the clinical response of the patient. Plasma EBV-DNA load decreased after treatment with GEM, which was also shown to be effective in the corresponding NPC02 PDX system. The patient died from aspiration pneumonia with septic shock 3 months after the last plasma EBV-DNA detection. Whole-body bone scan before B. and after C. GEM treatment at 3-month intervals, revealing stable disease (hot spots in right chest wall ribs in (C) are due to trauma). Abbreviations: PULB, cisplatin, tegafur/uracil, leucovorin, and bleomycin; MUB, methotrexate, tegafur/uracil, and bleomycin; FMP, tegafur/uracil, mitomycin-C, and cisplatin; FM, tegafur/uracil and mitomycin-C; VP+U, etoposide and tegafur/uracil; FA, tegafur/uracil and doxorubicin.

Furthermore, a histological examination of hematoxylin and eosin (H\&E)-stained tumors after treatment showed fewer viable tumor cells in the ganciclovir-containing three-drug group than in the gemcitabine + valproic acid group. EBER in situ hybridization staining also showed fewer EBER-positive cells in the three-drug treatment group (Fig. 6C and 6D). These results suggest that the ganciclovir-containing three-drug regimen is the best combination among those tested.

\section{DISCUSSION}

$\mathrm{NPC}$ is an EBV-related cancer in which the tumor microenvironment has a crucial role in tumor progression. Here, using metastatic tumors, which have greater engrafting potential than primary site tumors in the PDX model [27], we established EBV-positive PDX lines from two patients with metastatic NPC, including one from a neck lymph node core biopsy sample. Maintaining these xenograft lines may help retain EBV in tumor cells, and subsequently transferring these xenografts to an in vitro culture system could enable the establishment of new EBV-positive NPC cell lines.
Our microarray analyses showed that the gene expression profile of the PDX tumor was highly similar to that of the parent tumor, confirming that the PDX system recapitulates features of the clinical sample. In our PDX drug-sensitivity screens, gemcitabine was the best candidate among the commonly used therapeutic agents tested. We further clinically validated drug sensitivity results obtained with cell line/PDX models in the original patient (Fig. 4), showing that gemcitabine exerted excellent anticancer effects in the corresponding patient, who had been heavily and unsuccessfully treated with a number of chemotherapeutic regimens. We also demonstrated that gemcitabine plays a decisive role in combination CVAT regimens (Fig. 5B). Although docetaxel was found to be superior to gemcitabine in our in vitro cell line-based drug-sensitivity screens, it showed limited efficacy in the PDX system owing to toxicity, which manifested as diarrhea. Mitomycin-C was also active in PDX screens and this activity was correlated with a positive clinical response to mitomycin-C, used as a third-line treatment, that was maintained for almost 1 year. The combination of HDAC-inhibitor (valproic acid) and antiviral agent (ganciclovir), which has shown 
(A)

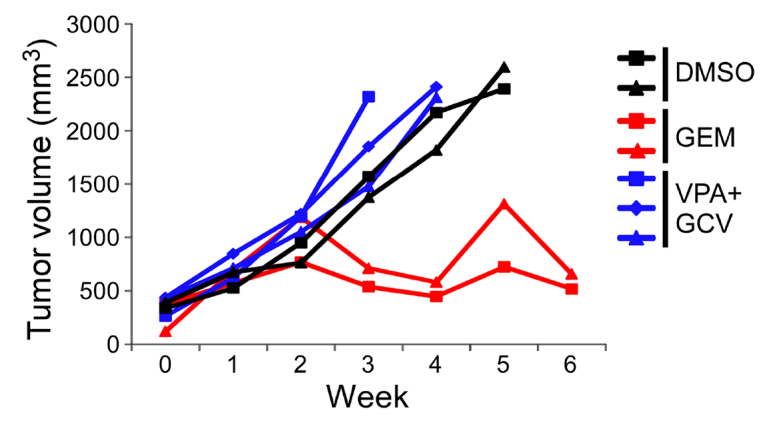

(B)

(8)

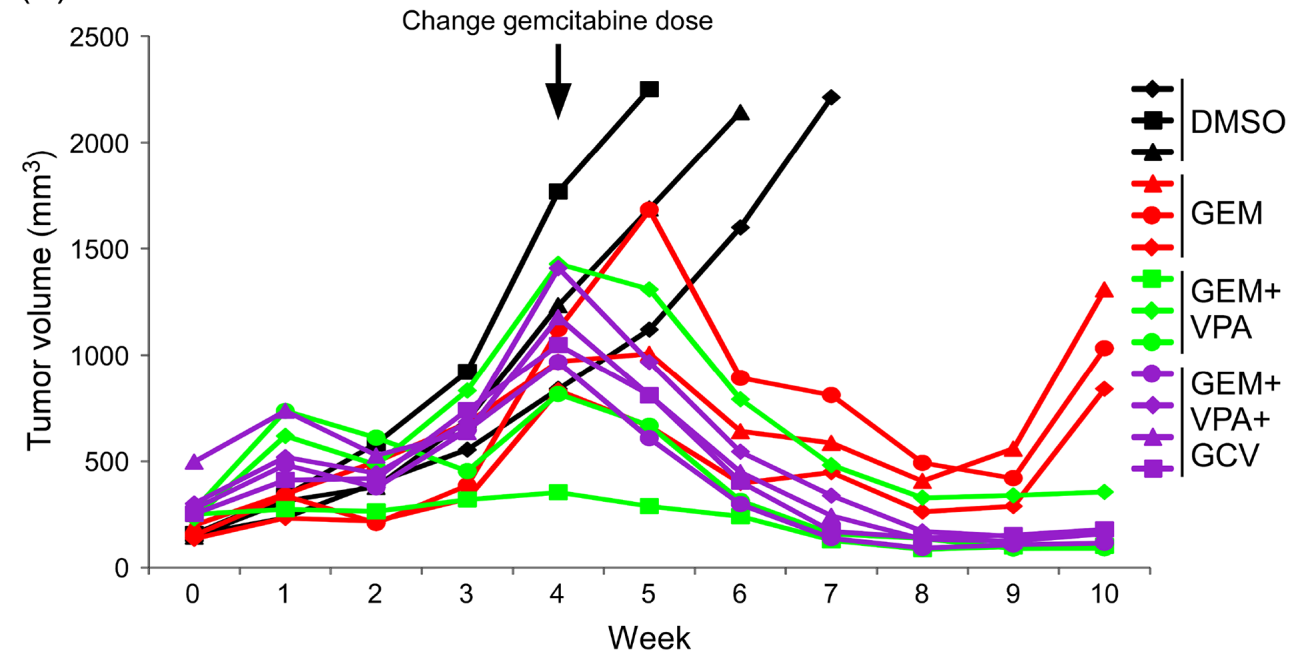

(C)

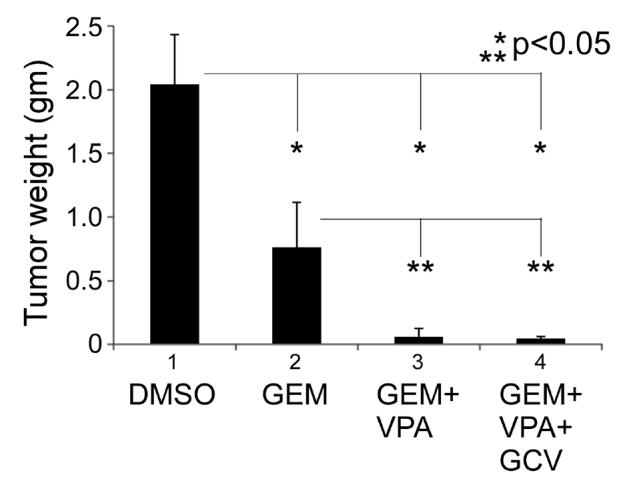

Figure 5: Tumor suppressive effects of different gemcitabine-based regimens in the PDX model. A. Valproic acid (VPA) combined with ganciclovir (GCV) had no antitumor effect in this PDX model. The effect of gemcitabine (GEM) alone (2 mg/kg) is shown for comparison. After the NPC02F3 PDX had been sub-implanted in NOD/SCID mice and the xenograft had reached a volume of approximately $1000 \mathrm{~mm}^{3}$, animals were randomized (2-3 tumor-bearing mice per group) and treated with the following dose schedule: DMSO $100 \mu$, 5 times/wk; GEM, $2 \mathrm{mg} / \mathrm{kg}, 5$ times/wk; VPA, $50 \mathrm{mg} / \mathrm{kg}, 5$ times/wk; and GCV, $50 \mathrm{mg} / \mathrm{kg}, 5$ times/wk. All chemicals were applied for 2 weeks every 3 weeks. B. Effects of GEM-based regimens in the PDX system. Low-dose GEM $(1 \mathrm{mg} / \mathrm{kg})$ given during the first 4 weeks of treatment showed little or no antitumor effect in GEM-containing groups. Starting on week 5, the GEM dose was increased to $2 \mathrm{mg} / \mathrm{kg}$. After subimplanted NPC02F5 PDX had reached a volume of approximately $500 \mathrm{~mm}^{3}$, animals were randomized (3-4 tumor-bearing mice per group) and treated with the following dose schedule: DMSO $100 \mu \mathrm{l}, 5$ times $/ \mathrm{wk}, 1 \mathrm{wk} / 3 \mathrm{wk} ; \mathrm{GEM}, 1 \mathrm{mg} / \mathrm{kg}, 5 \mathrm{times} / \mathrm{wk}, 1 \mathrm{wk} / 3 \mathrm{wk}$; VPA, $50 \mathrm{mg} / \mathrm{kg}$, 5 times/wk, $1 \mathrm{wk} / 3 \mathrm{wk}$ for GEM + VPA group and $2 \mathrm{wk} / 3 \mathrm{wk}$ for GEM + VPA + GCV group; and GCV, $50 \mathrm{mg} / \mathrm{kg}, 5$ times/wk, $2{ }^{\text {nd }} \mathrm{wk} / 3 \mathrm{wk}$. At the beginning of the fifth week, all treatment schedules were reset and the same dose schedule was applied except the GEM dose was increased to $2 \mathrm{mg} / \mathrm{kg}$. C. Xenograft weights for different treatment regimens in (B) 
(A)

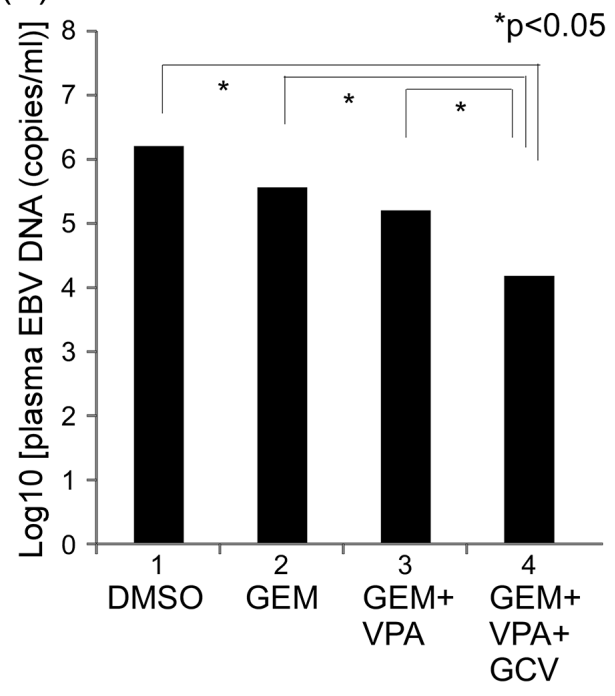

(B)

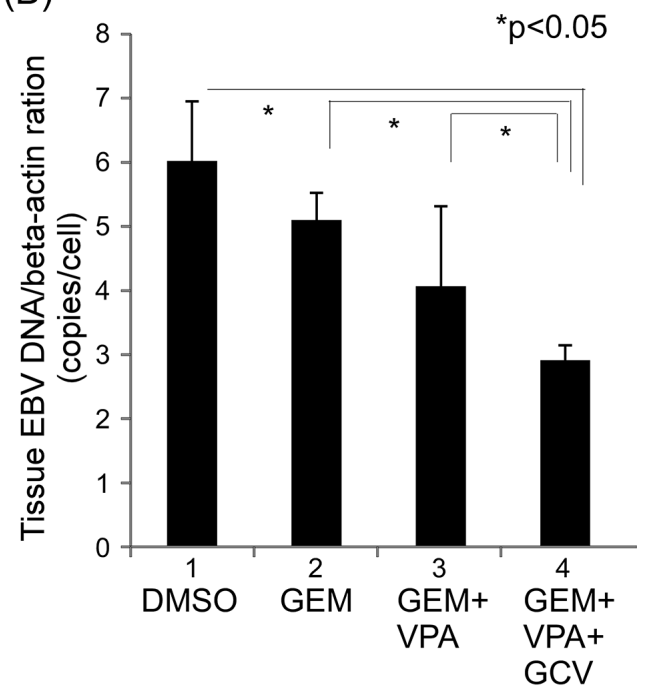

(C)
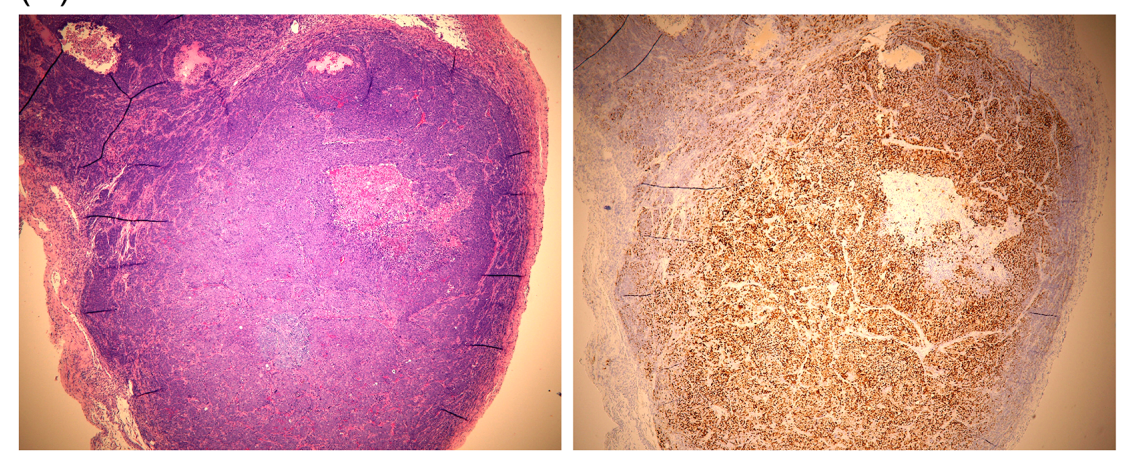

(D)
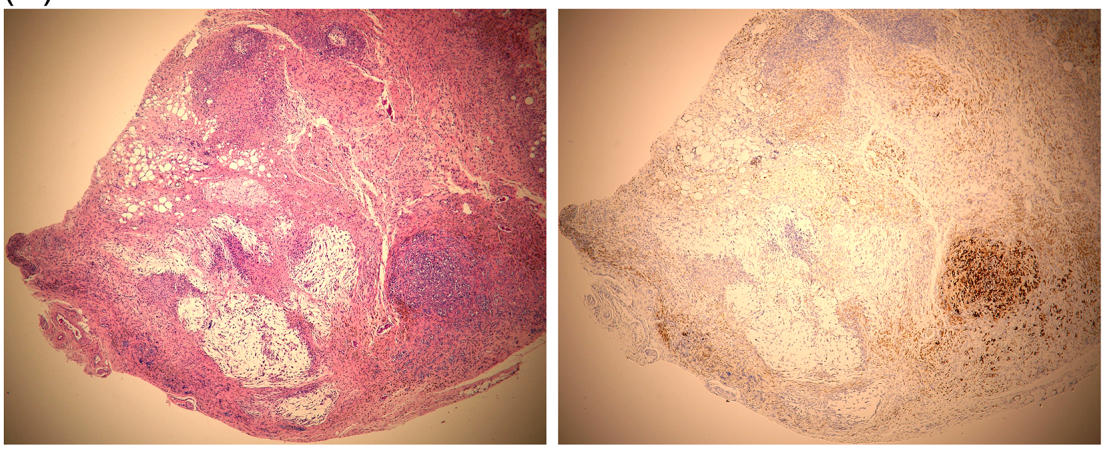

Figure 6: EBV activity decreased in the ganciclovir-containing treatment group. A. Plasma EBV-DNA load and B. xenograft tissue viral concentration. NPC02F6 PDX were used in these studies with two cycles of the same drug schedule as shown in Figure 5B. Tumor sections from the ganciclovir (GEV) + valproic acid (VPA) treatment group C. and gemcitabine (GEM) + VPA + GCV treatment group D. Left: H\&E staining; right: EBER staining. Original magnification, 40x.

some efficacy in certain lymphoma models [28], was not effective in our NPC PDX model (Fig. 5A).

The anticancer drugs tested demonstrate efficacy in clinical practice, but their response rates are variablegenerally less than $50 \%$ [29]. The high tumor heterogeneity within and between tumors in a given patient and between tumors in different patients is likely a contributing factor to this variability. Notable in this context, the current PDX was derived from tumors from only two cases amplified in
NOD/SCID mice. Taken together, these observations may help explain why some commonly used chemotherapeutic agents were ineffective in our PDX system.

The PDX system has previously been applied for drug-sensitivity screening and target-gene identification $[8,30,31]$. The true value of the PDX model for drug sensitivity testing lies in its role as a bridge between cell line studies and clinical tests, but it still has limitations, including restrictions associated with surgical/biopsy 
tissue processing, technically complicated orthotopic implantation, limited availability of original source material, low take out rate, and the time-consuming nature of xenograft growth [32, 33]. Different animal species also have different drug sensitivities and/or tolerances; for example, docetaxel has greater gastroenteritis side effects in NOD/SCID mice than in humans. Another potential weak point is the immunocompromised background of mice, which could limit its utility, since some inflammation/immune-related phenomena may be abolished in this system [8]. We also observed that some hematopoietic/macrophage-related genes were expressed at a low level in a PDX background. A high percentage of lymphoma (32.5\%) in PDX attributable the lymphomagenesis background of NOD/SCID mice has also recently been reported [34]. In this context, approximately $5 \%$ of our PDX-positive mice showed contamination by this spontaneous lymphomagenesis, with rapid progression of the tumor after passage.

EBV is considered a therapeutic target in EBV-related cancers, including lymphoma and NPC [25]. Valproic acid, a short chain fatty acid used clinically as an anti-epilepsy drug, could have some HDAC-inhibitory effects [35]. This HDAC inhibition may sensitize cancer cells to the effects of gemcitabine, and the combination of the drugs in these two categories has been shown to exert synergistic anticancer effects [22]. Our current PDX model, which revealed the key role of gemcitabine in combination regimens, also confirmed this concept. More recently, CVAT composed of a standard chemotherapeutic agent (gemcitabine), an HDAC inhibitor (valproic acid), and an antiviral agent (ganciclovir) has shown promising anticancer effects in refractory NPC patients [26]. Although the similar tumor control achieved with this three-drug combination regimen and the two-drug gemcitabine + valproic acid combination would seem to raise questions about the role of ganciclovir (Fig. 5), a detailed analysis demonstrated diminished EBVrelated activity in the ganciclovir-containing, three-drug treatment group, as evidenced by lower plasma EBV-DNA load, decreased tumor EBV concentration, and fewer viable EBV-positive tumor cells (Fig. 6). These results suggest that the ganciclovir-containing three-drug regimen is the best combination among those tested. Although chemotherapyrelated toxicity observed in patients treated with this ganciclovir-containing three-drug regimen is reported to be mild [26], additional clinical trials will be required to further confirm the reduced side effects, as well as the excellent tumor control efficacy, of this three-drug combination CVAT.

\section{MATERIALS AND METHODS}

\section{Materials}

Cisplatin, gemcitabine, docetaxel, etoposide, 5-fluorouracil (fluorouracil), mitomycin-C, valproic acid, and ganciclovir were purchased from Sigma Chemical Co (St. Louis, MO).

\section{Cell growth assay}

C666-1 cells were grown in RPMI medium containing $10 \%$ fetal bovine serum (FBS). Cells were plated at $5 \times 10^{4}$ cells/well in 24-well plates and incubated with or without different concentration of tested drugs for 6 days. Cell growth was assessed using the MTT (3-\{4,5-dimethylthiazol-2-yl $\}-2$, 5-diphenyltetrazolium bromide) assay [36]. At the end of studies, $50 \mu \mathrm{l}$ of a $5-\mathrm{mg} / \mathrm{mL}$ MTT solution was added to each well containing $500 \mu \mathrm{l}$ of medium, and plates were incubated for 3 hours; $500 \mu \mathrm{l}$ of isopropyl alcohol was then added to dissolve the reduced formazan product. The absorbance of each well was measured at a wavelength of $590 \mathrm{~nm}$ in a DU 640B spectrophotometer (Beckman, Fullerton, CA) according to the manufacturer's protocol. Values presented in figures are mean $\mathrm{OD}_{590} \pm \mathrm{SD}$ from at least three independent reaction wells.

\section{Animal studies}

All experiments involving laboratory animals were done in accordance with the Guideline for Animal Experiments of Chang Gung Memorial Hospital and were approved by the Animal Research Committee at Chang Gung Memorial Hospital.

The EBV-expressing NPC C666-1 cell line was used as a positive control. The cancer cells were harvested, washed twice with phosphate-buffed saline (PBS), and resuspended at a final concentration of $5 \times 10^{6}$ cells $/ \mathrm{mL}$ in Matrigel (BD Biosciences, San Jose, CA) containing basement membrane components. Then, $5 \times 10^{5}$ cells (100 $\mu \mathrm{L}$ per site) were subcutaneously injected into the flanks of 4-6-week-old male NOD/SCID mice (BioLASCO, Taiwan). Tumor development was confirmed within 2-3 weeks after injection of the same number of cell sub-clones. Treatment with different chemicals was initiated at the same time. Tumor dimensions were measured twice a week with calipers, and tumor volume was calculated with the formula, tumor volume $\left(\mathrm{mm}^{3}\right)=$ tumor length $(\mathrm{mm}) \times[\text { tumor width }(\mathrm{mm})]^{2} \times 0.5$. Tumors were harvested for further analysis. Three to five mice for each group (with or without chemical treatment) were used. Mice were sacrificed 3 months after chemical injection or earlier if tumors reached a size greater than $2000 \mathrm{~mm}^{3}$, body weight loss exceeded $20 \%$, mice were unable to maintain their normal food and water intake for 3 days, had micturition or defecation difficulties, or other conditions that would violate humane treatment regulations.

\section{Statistical analysis}

Cell line and tumor weight studies data are presented as means \pm SD. Final tumor volumes were compared using a two-tailed analysis of variance (ANOVA). 


\section{Patient enrollment}

Two biopsy-proven NPC patients with local recurrence or distant metastasis were enrolled between July 2013 and June 2014. Written informed consent approved by the Institutional Review Board of Chang Gung Memorial Hospital was obtained from these two participating patients. The recurrent/metastatic tissues were engrafted subcutaneously into NOD/SCID mice.

\section{PDX study}

Local recurrent/metastatic NPC tumor samples were obtained from patients undergoing surgical resection or biopsy. Each sample was immediately cut into small pieces $\left(25-30 \mathrm{~mm}^{3}\right)$ in PBS containing $200 \mathrm{U} / \mathrm{mL}$ penicillin and $200 \mu \mathrm{g} / \mathrm{mL}$ streptomycin and implanted subcutaneously in the flank region of anesthetized, 4-6-week-old male NOD/ SCID mice [32]. In the first round, two mice were each implanted with two pieces of tumor, where tumor volume permitted. Tumor size was measured twice per week with calipers, and the relative tumor volume was calculated. After the xenograft reached $\sim 1 \mathrm{~cm}^{3}$ in size, it was excised and sub-implanted into the next passage of mice. The PDX used in these studies were from the first eight passages of each line.

\section{Microarray analysis of tumor and xenograft}

Total RNA was extracted from cells using an RNeasy Mini kit (Qiagen, Valencia, CA) and evaluated by microarray analysis using the Human Whole Genome OneArray v6 (Phalanx Biotech Group, Taiwan), containing 32,679 DNA oligonucleotide probes, each of which is a 60 -mer designed in the sense direction. Among the probes, 31,741 corresponded to annotated genes in RefSeq v51 and Ensembl v65 database, and 938 corresponded to control probes. Fluorescent aRNA targets were prepared from $1 \mu \mathrm{g}$ total RNA samples using a OneArray ${ }^{\circledR}$ Amino Allyl aRNA Amplification Kit (Phalanx Biotech Group, Taiwan) and Cy5 dyes (Amersham Pharmacia, Piscataway, NJ). Fluorescent targets were hybridized to the Human Whole Genome OneArray ${ }^{\circledR}$ in Phalanx hybridization buffer using the Phalanx Hybridization System. After hybridization at $50^{\circ} \mathrm{C}$ for 16 hours, non-specific binding targets were removed using three different wash steps, and the slides were dried by centrifugation and scanned with an Axon 4000B Scanner (Molecular Devices, Sunnyvale, CA). The Cy5 fiuorescence intensity of each spot was analyzed using GenePix 4.1 software (Molecular Devices).

Signal intensity values for each spot were imported into the Rosetta Resolver System ${ }^{\circledR}$ (Rosetta Biosoftware) for data analysis. The error model of the Rosetta Resolver System, which is able to remove both systematic and random errors from the data, was used to filter out spots for which the flag value was less than 0. Spots that passed all criteria were normalized using the $50 \%$ media scaling normalization method. Reproducibility among technical repeat data was tested by calculating the Pearson correlation coefficient ( $\mathrm{R}$ value $>0.975$ ). Normalized spot intensities were transformed to $\log _{2}$ ratios of gene expression between control and treatment groups. Spots with a $\log _{2}$ ratio $\geq 1$ or $\leq-1$ and a $P$-value $<0.05$ were included in further analyses.

\section{Drug sensitivity tests in the PDX model}

After tumors had been sub-implanted in NOD/ SCID mice and xenograft had reached a volume of approximately $150 \mathrm{~mm}^{3}$, animals were randomized (3-5 mice with tumors on the right flank per group) and various drugs, including cisplatin, mitomycin- $\mathrm{C}$, fluorouracil, etoposide, gemcitabine, docetaxel, valproic acid, and ganciclovir, were administered via intraperitoneal injection. The following dose schedules were used: cisplatin, $4 \mathrm{mg} / \mathrm{kg}, 1$ time/wk; mitomycin-C, $3 \mathrm{mg} / \mathrm{kg}, 2$ times/wk; fluorouracil, $20 \mathrm{mg} / \mathrm{kg}, 1 \mathrm{time} / \mathrm{wk}$; etoposide, $12 \mathrm{mg} / \mathrm{kg}, 3$ times/wk; gemcitabine, $2 \mathrm{mg} / \mathrm{kg}$, 5 times/wk; docetaxel, $1 \mathrm{mg} / \mathrm{kg}, 5$ times/wk; valproic acid, $50 \mathrm{mg} / \mathrm{kg}, 5$ times/wk; and ganciclovir, $50 \mathrm{mg} / \mathrm{kg}$, 5 times/wk.

Mice xenografted with the EBV-positive cell line C666-1 served as a control. Final tumor volumes were compared using a two-tailed ANOVA, adjusted for multiple comparisons. A ranked list of effective treatments was provided to the attending physician, who then selected the patient treatment.

\section{EBV-DNA detection}

DNA was extracted from plasma/tissue as described previously $[6,32]$. Briefly, $10-\mathrm{mL}$ samples of peripheral blood were collected in EDTA-treated tubes and centrifuged at $1000 \mathrm{x}$ g for 15 minutes. Plasma/ tissue DNA was extracted using a QIAamp DNA Blood MiniKit (Qiagen, Valencia, CA). About 500-1000 $\mu \mathrm{L}$ of each sample per column (supplied in the QIAamp kit) was used for DNA extraction. DNA was eluted from each column with $80 \mu \mathrm{L}$ distilled water [4]. EBV-DNA concentrations were measured by real-time quantitative polymerase chain reaction (PCR) of the Bam HI-W region of the EBV genome [32]. Primer and probe sequences, including the dual fluorescence-labeled oligomer, and the detailed procedure have been described previously [6]. The relative EBV-DNA concentration in tissue was expressed as EBV-DNA copies/ $\beta$-actin gene copies in the same tested samples.

\section{Detection of EBV-encoded small RNAs}

Paraffin-embedded sections from patient tumors or mice xenografts were used for detection of EBER (EBV-encoded small RNAs) by fluorescence in situ hybridization. Tissue sections were deparaffinized and 
pretreated with proteinase $\mathrm{K}$ for 10 minutes and then incubated with a fluorescein-conjugated EBER DNA probe (Leica Biosystems, Newcastle, UK) at $37^{\circ} \mathrm{C}$ for 2 hours. The sections were rinsed in water and incubated with horseradish peroxidase-conjugated anti-fluorescein antibody for 15 minutes before addition of fresh DAB (3,3-diaminobenzidine) substrate to produce an alcoholinsoluble brown intranuclear stain in EBV-positive cells.

\section{Abbreviations}

5-FU, fluorouracil; EBV, Epstein Barr virus; EBER, EBV-encoded small RNAs; CVAT, cytolytic viral activation therapy; GCV, ganciclovir; GEM, gemcitabine; HDAC, histone deacetylase; NOD/SCID, nonobese diabetic/severe combined immunodeficiency; NPC, nasopharyngeal carcinoma; PDX, patient-derived xenograft; SCID, severe combined immunodeficiency; VPA, valproic acid.

\section{ACKNOWLEDGMENTS AND FUNDING}

We thank the staff at the Laboratory Animal Center, Chang Gung Memorial Hospital, Linkou, Taoyuan, Taiwan, for critical animal care.

This work was supported by grants from Chang Gung Memorial Hospital (CMRPG3C1931, CMRPG3C1932), and the Ministry of Science and Technology, Taiwan (MOST103-2314-B-182-041, MOST104-2314-B-182-036).

\section{CONFLICTS OF INTEREST}

The authors of this manuscript have nothing no conflicts of interest to declare.

\section{REFERENCES}

1. Yu MC, Yuan JM. Epidemiology of nasopharyngeal carcinoma. Seminars in cancer biology. 2002; 12:421-429.

2. Shanmugaratnam K, Sobin LH. The World Health Organization histological classification of tumours of the upper respiratory tract and ear. A commentary on the second edition. Cancer. 1993; 71:2689-2697.

3. Chang YS, Tyan YS, Liu ST, Tsai MS, Pao CC. Detection of Epstein-Barr virus DNA sequences in nasopharyngeal carcinoma cells by enzymatic DNA amplification. Journal of clinical microbiology. 1990; 28:2398-2402.

4. Lin JC, Wang WY, Chen KY, Wei YH, Liang WM, Jan JS, Jiang RS. Quantification of plasma Epstein-Barr virus DNA in patients with advanced nasopharyngeal carcinoma. The New England journal of medicine. 2004; 350:2461-2470.

5. Jin Y, Cai XY, Cai YC, Cao Y, Xia Q, Tan YT, Jiang WQ, Shi YX. To build a prognostic score model containing indispensible tumour markers for metastatic nasopharyngeal carcinoma in an epidemic area. European Journal of Cancer. 2012; 48:882-888.

6. Hsu CL, Chang KP, Lin CY, Chang HK, Wang $\mathrm{CH}$, Lin TL, Liao CT, Tsang NM, Lee LY, Chan SC, Ng SH, Li HP, Chang YS, Wang HM. Plasma epstein-barr virus DNA concentration and clearance rate as novel prognostic factors for metastatic nasopharyngeal carcinoma. Head \& neck. 2012; 34:1064-1070.

7. Hsu CL, Chan SC, Chang KP, Lin TL, Lin CY, Hsieh CH, Huang SF, Tsang NM, Lee LY, Ng SH, Wang HM. Clinical scenario of EBV DNA follow-up in patients of treated localized nasopharyngeal carcinoma. Oral oncology. 2013; 49:620-625.

8. Tentler JJ, Tan AC, Weekes CD, Jimeno A, Leong S, Pitts TM, Arcaroli JJ, Messersmith WA, Eckhardt SG. Patient-derived tumour xenografts as models for oncology drug development. Nature reviews Clinical oncology. 2012; 9:338-350.

9. Fichtner I, Rolff J, Soong R, Hoffmann J, Hammer S, Sommer A, Becker M, Merk J. Establishment of patientderived non-small cell lung cancer xenografts as models for the identification of predictive biomarkers. Clinical cancer research: an official journal of the American Association for Cancer Research. 2008; 14:6456-6468.

10. Sriuranpong V, Mutirangura A, Gillespie JW, Patel V, Amornphimoltham P, Molinolo AA, Kerekhanjanarong V, Supanakorn S, Supiyaphun P, Rangdaeng S, Voravud N, Gutkind JS. Global gene expression profile of nasopharyngeal carcinoma by laser capture microdissection and complementary DNA microarrays. Clinical cancer research: an official journal of the American Association for Cancer Research. 2004; 10:4944-4958.

11. Cabelguenne A, Blons $\mathrm{H}$, de Waziers I, Carnot F, Houllier AM, Soussi T, Brasnu D, Beaune P, Laccourreye O, Laurent-Puig P. p53 alterations predict tumor response to neoadjuvant chemotherapy in head and neck squamous cell carcinoma: a prospective series. Journal of clinical oncology: official journal of the American Society of Clinical Oncology. 2000; 18:1465-1473.

12. Fu X, Guadagni F, Hoffman RM. A metastatic nudemouse model of human pancreatic cancer constructed orthotopically with histologically intact patient specimens. Proceedings of the National Academy of Sciences of the United States of America. 1992; 89:5645-5649.

13. Kim MP, Evans DB, Wang H, Abbruzzese JL, Fleming JB, Gallick GE. Generation of orthotopic and heterotopic human pancreatic cancer xenografts in immunodeficient mice. Nature protocols. 2009; 4:1670-1680.

14. Villarroel MC, Rajeshkumar NV, Garrido-Laguna I, De Jesus-Acosta A, Jones S, Maitra A, Hruban RH, Eshleman JR, Klein A, Laheru D, Donehower R, Hidalgo M. Personalizing cancer treatment in the age of global genomic analyses: PALB2 gene mutations and the response to DNA damaging agents in pancreatic cancer. Molecular cancer therapeutics. 2011; 10:3-8. 
15. Gressette M, Verillaud B, Jimenez-Pailhes AS, Lelievre H, Lo KW, Ferrand FR, Gattolliat CH, Jacquet-Bescond A, Kraus-Berthier L, Depil S, Busson P. Treatment of nasopharyngeal carcinoma cells with the histone-deacetylase inhibitor abexinostat: cooperative effects with cis-platin and radiotherapy on patient-derived xenografts. PloS one. 2014; 9:e91325.

16. Cheung CC, Chung GT, Lun SW, To KF, Choy KW, Lau KM, Siu SP, Guan XY, Ngan RK, Yip TT, Busson P, Tsao SW, Lo KW. miR-31 is consistently inactivated in EBV-associated nasopharyngeal carcinoma and contributes to its tumorigenesis. Molecular cancer. 2014; 13:184.

17. Young LS, Rickinson AB. Epstein-Barr virus: 40 years on. Nature reviews Cancer. 2004; 4:757-768.

18. Kenney SC, Mertz JE. Regulation of the latent-lytic switch in Epstein-Barr virus. Seminars in cancer biology. 2014; 26:60-68.

19. Feng WH, Hong G, Delecluse HJ, Kenney SC. Lytic induction therapy for Epstein-Barr virus-positive B-cell lymphomas. Journal of virology. 2004; 78:1893-1902.

20. Fu DX, Tanhehco Y, Chen J, Foss CA, Fox JJ, Chong JM, Hobbs RF, Fukayama M, Sgouros G, Kowalski J, Pomper MG, Ambinder RF. Bortezomib-induced enzymetargeted radiation therapy in herpesvirus-associated tumors. Nature medicine. 2008; 14:1118-1122.

21. Westphal EM, Blackstock W, Feng W, Israel B, Kenney SC. Activation of lytic Epstein-Barr virus (EBV) infection by radiation and sodium butyrate in vitro and in vivo: a potential method for treating EBV-positive malignancies. Cancer research. 2000; 60:5781-5788.

22. Feng WH, Kenney SC. Valproic acid enhances the efficacy of chemotherapy in EBV-positive tumors by increasing lytic viral gene expression. Cancer research. 2006; 66:8762-8769.

23. Roychowdhury S, Peng R, Baiocchi RA, Bhatt D, Vourganti S, Grecula J, Gupta N, Eisenbeis CF, Nuovo GJ, Yang W, Schmalbrock P, Ferketich A, Moeschberger M, Porcu P, Barth RF, Caligiuri MA. Experimental treatment of Epstein-Barr virus-associated primary central nervous system lymphoma. Cancer research. 2003; 63:965-971.

24. Meng Q, Hagemeier SR, Fingeroth JD, Gershburg E, Pagano JS, Kenney SC. The Epstein-Barr virus (EBV)encoded protein kinase, EBV-PK, but not the thymidine kinase (EBV-TK), is required for ganciclovir and acyclovir inhibition of lytic viral production. Journal of virology. 2010; 84:4534-4542.

25. Mentzer SJ, Fingeroth J, Reilly JJ, Perrine SP, Faller DV. Arginine butyrate-induced susceptibility to ganciclovir in an Epstein-Barr-virus-associated lymphoma. Blood cells, molecules \& diseases. 1998; 24:114-123.

26. Wildeman MA, Novalic Z, Verkuijlen SA, Juwana H, Huitema AD, Tan IB, Middeldorp JM, de Boer JP, Greijer AE. Cytolytic virus activation therapy for
Epstein-Barr virus-driven tumors. Clinical cancer research: an official journal of the American Association for Cancer Research. 2012; 18:5061-5070.

27. DeRose YS, Wang G, Lin YC, Bernard PS, Buys SS, Ebbert MT, Factor R, Matsen C, Milash BA, Nelson E, Neumayer L, Randall RL, Stijleman IJ, Welm BE, Welm AL. Tumor grafts derived from women with breast cancer authentically reflect tumor pathology, growth, metastasis and disease outcomes. Nature medicine. 2011; 17:1514-1520.

28. Ghosh SK, Perrine SP, Williams RM, Faller DV. Histone deacetylase inhibitors are potent inducers of gene expression in latent EBV and sensitize lymphoma cells to nucleoside antiviral agents. Blood. 2012; 119:1008-1017.

29. Ali H, al-Sarraf M. Chemotherapy in advanced nasopharyngeal cancer. Oncology (Williston Park). 2000; 14:1223-1230.

30. Einarsdottir BO, Bagge RO, Bhadury J, Jespersen H, Mattsson J, Nilsson LM, Truve K, Lopez MD, Naredi P, Nilsson O, Stierner U, Ny L, Nilsson JA. Melanoma patient-derived xenografts accurately model the disease and develop fast enough to guide treatment decisions. Oncotarget. 2014; 5:9609-9618.

31. Dobbin ZC, Katre AA, Steg AD, Erickson BK, Shah MM, Alvarez RD, Conner MG, Schneider D, Chen D, Landen CN. Using heterogeneity of the patient-derived xenograft model to identify the chemoresistant population in ovarian cancer. Oncotarget. 2014; 5:8750-8764.

32. Moro M, Bertolini G, Tortoreto M, Pastorino U, Sozzi G, Roz L. Patient-Derived Xenografts of Non Small Cell Lung Cancer: Resurgence of an Old Model for Investigation of Modern Concepts of Tailored Therapy and Cancer Stem Cells. Journal of Biomedicine and Biotechnology. 2012; 2012. pii:568567.

33. Whittle JR, Lewis MT, Lindeman GJ, Visvader JE. Patientderived xenograft models of breast cancer and their predictive power. Breast cancer research. 2015; 17:17.

34. Zhang L, Liu Y, Wang X, Tang Z, Li S, Hu Y, Zong X, Wu X, Bu Z, Wu A, Li Z, Li Z, Huang X, Jia L, Kang Q, Liu $Y$, et al. The extent of inflammatory infiltration in primary cancer tissues is associated with lymphomagenesis in immunodeficient mice. Scientific Reports. 2015; 5:9447.

35. Chateauvieux S, Morceau F, Dicato M, Diederich M. Molecular and Therapeutic Potential and Toxicity of Valproic Acid. Journal of Biomedicine and Biotechnology. 2010; 2010. pii: 479364.

36. Hsu CL, Chen YL, Ting HJ, Lin WJ, Yang Z, Zhang Y, Wang L, Wu CT, Chang HC, Yeh S, Pimplikar SW, Chang C. Androgen receptor (AR) NH2- and $\mathrm{COOH}-$ terminal interactions result in the differential influences on the AR-mediated transactivation and cell growth. Molecular endocrinology. 2005; 19:350-361. 